\title{
Maturation de Form@sup : principes et instruments
}

\author{
Marianne Poumay \\ LabSET - Université de Liège, BELGIQUE \\ m.poumay@ulg.ac.be
}

\section{Compte rendu d'expérience}

\section{Résumé}

Sans en reprendre la description détaillée, le présent article propose une analyse critique des options prises dans Form@sup, formation postmaîtrise d'une année pour les enseignants du supérieur désireux de s'interroger sur leur pratique professionnelle en développant leur cours en ligne. L'article justifie les fondements théoriques de ce dispositif et souligne les difficultés rencontrées dans la mise en œuvre de chacun de ses principes de base. Il présente ensuite les options prises pour 2005-2006, insistant sur l'influence du courant de professionnalisation de l'enseignement dans le supérieur. Par ses exemples concrets, il vise aussi à susciter la réflexion et l'échange de pratiques parmi les équipes de « développeurs instructionnels ».

\section{Summary}

Without resuming its detailed description, the present article proposes a critical analysis of the options taken in Form@sup, a one-year postgraduate degree for higher education teachers who want to question their professional practice through the development of their online course. The article justifies the theoretical backgrounds of this training program and underlines the difficulties encountered in carrying out each of its basic principles. It then presents the decisions made for 2005-2006, insisting on the influence of the Scholarship of Teaching and Learning movement. Through its concrete examples, it also aims at fostering reflection and exchange of practice amongst the instructional developers teams.

CAuteur(s). Cette œuvre, disponible à http://ritpu.ca/IMG/pdf/RITPU_POUMAY02_3-2.pdf, est mise à disposition selon les termes de la licence Creative Commons Attribution - Pas de Modification 2.5 Canada : http://creativecommons.org/licences/by-nd/2.5/ca/deed.fr 


\section{Introduction}

Organisée à l’Université de Liège (Belgique), Form@sup est une formation postmaîtrise d'une année (60 ECTS) qui vise à aider les collègues de l'enseignement supérieur à modifier leur pratique professionnelle. Pour chaque participant, les piliers de cette formation sont une réflexion approfondie sur son enseignement, une recherche personnelle et la transformation du cours dont il a la charge pour y introduire soit l'apprentissage basé sur les problèmes (APP ou PBL), soit l'eLearning.

Le présent article ne peut se comprendre sans une prise de connaissance du dispositif dont il analyse de façon critique l'option eLearning ${ }^{1}$. Ce dispositif Form@sup a été décrit dans l'article intitulé « Pour professionnaliser le métier d'enseignant du supérieur: le Master complémentaire Form@sup » (Poumay, 2007). Nous y renvoyons donc le lecteur.

Complémentairement, après avoir rapidement brossé son contexte, nous analysons ici les options prises dans Form@sup, nous en expliquons les fondements théoriques et soulignons dans des encadrés les difficultés rencontrées, non pas de manière exhaustive, mais plutôt en nous centrant sur les principaux écueils. Les décisions de régulation sont explicitées, pour guider des équipes qui souhaiteraient transférer ce type de réflexion à leur propre dispositif de formation ou simplement tirer parti de nos tâtonnements.

\section{Le contexte de Form@sup}

Cheval de Troie de la pédagogie, le déclencheur de Form@sup est sans conteste l'eLearning. En Europe, l'un des défis actuels de l'éducation est le développement d'une masse critique de cours à distance $^{2}$ de qualité qui permette la dissémination de bonnes pratiques en langues locales et accessibles à tous. En Belgique, l'offre de formation à distance est encore plutôt réduite alors que de nombreux acteurs souhaitent avoir accès à des solutions flexibles. Des mesures concrètes ont ce- pendant vu le jour ces dernières années. Ainsi, les grandes universités se sont dotées de centres ou cellules internes qui, parmi leurs mandats, aident les enseignants dans le portage en ligne de leurs cours. Les gouvernements successifs ont eux aussi lancé des projets fédérateurs comme le portail wallon de l'eLearning $^{3}$ ou le projet Formadis ${ }^{4}$. Parallèlement à ces mesures, d'autres initiatives gouvernementales visent l'accessibilité, par exemple en formant annuellement 20000 demandeurs d'emploi aux manipulations de base d'outils comme Internet ou un traitement de texte (projet $\mathrm{PMTIC}^{5}$ ), ceci grâce à trois modules de formation progressifs et à un dispositif basé sur la proximité.

Issu de ce contexte propice aux innovations technologiques en pédagogie, le LabSET est au service de tout encadrant (professeur, formateur) désireux d'améliorer son enseignement par le biais de supports Web. Au sein de l'Université de Liège, il compte aujourd'hui plus de 30 personnes qui, outre les projets de recherche, accompagnent annuellement une quarantaine d'enseignants dans la transformation et le portage à distance de leurs propres cours, touchant plusieurs milliers d'utilisateurs finaux. Utiliser l'eLearning comme une porte d'entrée vers la modification des pratiques pédagogiques est généralement bien accepté par les enseignants, qui reconnaissent volontiers avoir beaucoup à apprendre en matière de maîtrise technologique... alors qu'ils sont moins ouverts si l'on aborde de front leurs méthodes d'enseignement ou leurs techniques d'évaluation.

Notre porte d'entrée eLearning n'avait de sens qu'assortie à notre présupposé de base: une formation en pédagogie de l'enseignement supérieur est susceptible de sensiblement améliorer la qualité de l'enseignement. Boice (2000, p. 12) constate que «la tradition, dans le monde académique, véhicule l'idée erronée que connaître sa matière entraîne savoir l'enseigner. Sous forme de formule, on écrirait bonne connaissance matière = bon enseignement». Form@sup lutte lui aussi 
contre cette égalité simpliste et souhaite outiller les enseignants tant dans leur pratique réflexive (Schön, 1983/1994) que dans leurs instruments pédagogiques et leurs maîtrises technologiques. Une seconde constatation de Boice (2000, p. 14), tout aussi triste, est également attaquée de front par Form@sup : «les campus ignorent les performances des jeunes enseignants pour autant qu'elles ne déclenchent pas une avalanche de plaintes d'étudiants; les jeunes enseignants sont abandonnés à leur sort de "nage ou coule", sans doute parce qu'ils devraient apprendre par eux-mêmes, ou qu'enseigner ne s'apprend pas. » Une offre de développement professionnel aide ces jeunes collègues $^{6}$ à se questionner et à progresser.

Pour bâtir le dispositif, l'équipe du LabSET s'est appuyée sur des principes pédagogiques socioconstructivistes, une veille permanente au travers de conférences et de nombreuses lectures, le courant de professionnalisation de l'enseignement supérieur initié par Boyer (1990) et les définitions de niveaux de professionnalisation de Kreber (2002). Nos propres pratiques antérieures (par exemple dans le projet Formadis, mentionné plus haut, qui a débuté avant Form@sup) et une étude sur les facteurs de succès de l'implantation de l'eLearning (Delfosse, Harmeling, Poumay et Leclercq, 2003) ont été la base de notre méthode "orientée projets ». Nous avons formalisé (Poumay, 2003) la plupart de ces principes dans une approche nommée «Construction de cours avec coaching étroit et formation intégrée ».

\section{Pourquoi un curriculum diplômant?}

Dans la majorité des pays européens, aucune stratégie nationale d'amélioration du professionnalisme dans l'enseignement supérieur n'est mise en œuvre. Pourtant, les défis sont grands. Par exemple, les taux d'échecs constatés sont très importants (Leclercq et al., 2003, p. 16). Diane Laurillard, décrivant le contexte du Royaume-Uni, lie clairement ce besoin de professionnalisme au besoin de valorisation des enseignants. Elle affirme (2002, p. 235) que nous devrions

"nous assurer que des procédures d'encouragement et de promotion récompensent l'excellence en enseignement (...). Si un soutien évident pour de telles mesures est constaté au niveau des cadres supérieurs, renforcé comme il l'est par les priorités nationales, alors la communauté académique commencera à croire que l'excellence en enseignement jouit d'un statut similaire à celui dont bénéficie l'excellence en recherche. »

Dans ce même sens, Lambert et Tice (1993, p. VIIIIX) constatent qu'aujourd'hui,

«les programmes de pointe ont des buts bien plus élevés: préparer la prochaine génération d'enseignants du supérieur à l'excellence à la fois en recherche et en enseignement (...) et contribuer à la modification des cultures de campus de façon à ce que celles-ci valorisent plus (et gratifient) l'excellence en enseignement. »

Form@sup offre la particularité d'être diplômant. À défaut de stratégie nationale, il contribue ainsi à la reconnaissance et à la valorisation des initiatives individuelles visant l'amélioration des pratiques d'enseignement. La reconnaissance du curriculum comme Master complémentaire dans la ligne du processus de Bologne était un signe de notre ministère de l'Éducation marquant l'importance de la pédagogie universitaire et de son déploiement en tant que discipline à part entière. Cette mesure entrera en vigueur en 2007.

De plus en plus, les évaluations internationales considéreront l'excellence pédagogique comme un critère digne de figurer parmi les indicateurs de la qualité d'une institution, au même rang que l'excellence en matière de recherche. La proportion 
d'enseignants qui possèdent un réel titre pédagogique pour enseigner leur discipline sera prise en compte dans ces jugements interinstitutionnels. Au niveau individuel, obtenir un titre pédagogique sera un moteur d'avancement, comme c'est déjà le cas dans certains pays comme l'Angleterre, le Sri Lanka ou la Suède.

Proposer un curriculum diplômant est donc stratégiquement un pas important vers la dissémination de pratiques positionnant la pédagogie comme un élément important pour l'efficacité des institutions d'enseignement et la reconnaissance des individus qui y consacrent l'énergie nécessaire à une année de formation complémentaire.

Les difficultés rencontrées à cette étape sont essentiellement d'ordre institutionnel et administratif.

Créer un diplôme constitue un processus lent, qui demande de passer par les arcanes du département, de la faculté et du conseil d'administration. Des tensions émergent des craintes de concurrence. Le projet se prépare environ deux ans avant de voir ses premiers inscrits.

Le bilinguisme d'un curriculum diplômant crée aussi des difficultés dues à la traduction des formulaires d'inscription à l'université, nécessaire présence de ces formulaires en ligne, demandes de bourses pour certains participants étrangers, traduction jurée des documents finalisant l'admission des candidats ou paiements retardés pour cause de mandats internationaux.

\section{Pourquoi sélectionner les participants sur projets?}

Form@sup a choisi de sélectionner les participants non pas par souci d'élitisme, mais bien parce que nos forces d'accompagnement ne sont pas illimitées.
Parmi les critères de sélection, on retiendra essentiellement la motivation des candidats (certains sont d'ailleurs invités à un entretien pour éclaircir ces motivations), l'avancement de leur réflexion et la faisabilité de leur projet (objectifs réalistes, matériel préexistant, temps disponible). Il arrive qu'un enseignant remplisse le formulaire de candidature puis, à la suite d'un entretien, décide de mûrir son projet durant une année avant de s'inscrire l'année suivante.

La sélection est au service de la qualité de la formation: elle offre à chacun des possibilités de discuter son projet et donc de voir si la formation lui convient et elle permet de ne pas engager l'équipe du LabSET dans un encadrement massif qu'elle ne pourrait assumer. Un groupe de 10 à 20 participants nous semble idéal pour assurer une qualité d'accompagnement et d'interaction en correspondance avec nos exigences de résultats.

En 2002, 11 participants ont été sélectionnés dans l'option eLearning ; 14 ont été sélectionnés en 2003 et 18 en 2004. Il est rare qu'un participant abandonne en cours de route, mais il arrive qu'il décide d'étaler son effort sur deux années académiques.

Les difficultés rencontrées ici sont d'ordre organisationnel: il nous faut imposer une rentrée de formulaires de candidatures en août, organiser un comité de sélection, dépouiller les candidatures, recevoir certains candidats ou organiser des conférences téléphoniques, se mettre d'accord sur des décisions finales et les communiquer aux candidats, le tout avant fin septembre. Cela représente une charge de travail supplémentaire et mobilise quatre personnes.

\section{Pourquoi une focalisation sur ces projets tout au long de la formation?}

Notre approche, apparemment centrée sur des projets d'eLearning, nécessite en fait une réflexion 
approfondie et constitue un cheval de Troie pour la révision globale $\mathrm{du}$ « design instructionnel ${ }^{8}$ » du cours de chaque participant. Le passage à l'eLearning est ici un prétexte à une refonte pédagogique visant la qualité ${ }^{9}$, quelles que soient les technologies employées. Nous profitons de l'effet décrit par Sanders (2001, p. 290) :

«[...] un intérêt de l'introduction des technologies dans l'enseignement a été l'ouverture de débats à propos de pédagogie [...]. Un effet secondaire est la double introduction de la technologie et de la pédagogie dans des environnements où elles n'avaient plus été discutées depuis bien longtemps. »

L'approche projet présente un autre avantage majeur : elle favorise grandement la motivation des participants qui voient se développer leur propre réalisation. Les trois types de perception influençant la motivation en contexte scolaire (Viau, 1994) sont tous trois largement positifs: le projet étant fonctionnel pour le participant, la perception de la valeur de l'activité est très haute; l'accompagnement rapproché rend la perception de sa compétence à l'accomplir très positive; la perception de la contrôlabilité du déroulement de la tâche et de ses conséquences est, elle aussi, très haute puisque chacun pose l'entièreté des choix concernant son propre cours.

Les difficultés sont ici plutôt d'ordre pédagogique: lorsqu'un curriculum fait une large place au projet personnel $\mathrm{du}$ participant, l'exigence de l'apprentissage just in time est très importante de la part des participants, qui visent l'utilité directe de chaque apprentissage à leur projet personnel, parfois aux dépens d'une attitude réflexive. Par ailleurs, il est peu aisé d'organiser des échanges d'expériences au sein $d^{\prime}$ 'un groupe dans lequel chacun se trouve à un stade différent de développement de son projet. Les accompagnateurs sont nécessaires pour faciliter la recherche d'intérêts croisés entre projets et générer des échanges toujours considérés par les participants comme enrichissants... mais pas forcément prioritaires.

À cette difficulté pédagogique s'ajoute une difficulté organisationnelle et financière : notre taux d'encadrement est supérieur à celui de la plupart des dispositifs conventionnels, l'encadrement des projets en est une des raisons.

\section{Pourquoi une pratique de méthodes actives et variées?}

Ce sont les arguments et principes pédagogiques issus des théories socioconstructivistes qui nous ont fait placer au centre de nos méthodes les activités, et plus particulièrement les activités collectives, comme puissant moteur de l'apprentissage. Pour illustrer l'importance d'un vécu actif et varié, nous mentionnerons ici quatre arguments et principes qui entrent bien en résonance avec le socioconstructivisme, mais sont peu souvent cités et illustrés : les principes d'isomorphisme visant un transfert à la pratique, d'extension de la zone proximale de développement pour un élargissement de l'univers des possibles, d'adaptation aux variations inter- et intraindividuelles (styles d'apprentissage changeants) et de déclenchement de la métacognition.

Principe d'isomorphisme. Un premier intérêt de cette variation d'activités est de pouvoir faire vivre aux enseignants ce que nous souhaitons (dans les principes en tout cas) qu'ils fassent vivre à leurs étudiants. Cet isomorphisme des méthodes donne plus de chances à certaines de nos pratiques d'être transférées en connaissance de cause dans celles que mettront en œuvre nos participants avec leurs propres étudiants. Non pas que nos pratiques soient exemplaires et méritent d'être transférées telles quelles, mais nous pensons qu'elles ont la richesse suffisante pour provoquer des débats et des prises de position. 
Principe d'extension de la zone proximale de développement. Un second avantage de ce vécu varié est qu'il étend chez chacun l'univers des possibles. Il nourrit l'imaginaire sur ce que pourraient être l'enseignement et l'apprentissage si l'on souhaitait les améliorer. Or, souvent, l'enseignant reste enfermé dans ses méthodes... tout simplement parce qu'il n'en connaît pas d'autres!

\section{Principe d'adaptation aux variations} interindividuelles ou enseignement différencié. Parmi les vécus proposés, certaines activités conviennent mieux que d'autres à certains points de matière, certaines parlent plus à certains enseignants à ce moment de leur questionnement, certaines semblent plus "praticables » grâce à leur facilité de mise en œuvre, certaines correspondent bien à la personnalité d'un enseignant, à ce qu'il voit comme étant ses forces ou à ses souhaits d'évolution. Proposer un vécu varié a donc plus de chances de rencontrer chaque participant à un moment de sa propre réflexion. Cela génère aussi une prise de conscience de l'intérêt de proposer une telle diversité à ses propres étudiants, pour les mêmes raisons. Avec Coffield, Moseley, Hall et Ecclestone (2004), nous rejoignons ici les considérations d'auteurs comme Entwistle, McCune et Walker (Approaches and study skills inventory for students, 2001), Sternberg (Thinking styles, 2001), Vermunt (Inventory of learning styles, 1994) ou, dans une moindre mesure, Allinson et Hayes (Cognitive style index, 1996), Herrmann (Brain dominance instrument, 1996), Honey et Mumford (Learning style questionnaire, 2000) ou encore Kolb (Learning style inventory, 1984, 1999), qui prônent la variation des activités pour tenir compte des styles d'apprentissage parfois changeants. Les outils développés par ces auteurs (indiqués en italique cidessus) ne doivent pas viser à catégoriser les apprenants dans des boîtes fermées, mais bien à mieux comprendre leur diversité et la façon dont ils s'adaptent en fonction de multiples variables. Ces styles étant multiples et changeants, y compris chez une même personne, varier les approches et les activités au sein d'un groupe donne un maximum de chances de faire écho aux préoccupations momentanées de chacun. En respectant les variations interindividuelles, nous rencontrons donc aussi un principe d'adaptation aux variations intraindividuelles.

\section{Principe de déclenchement de la métacognition.}

Pour Kelly (2005), « le premier pas est sans doute de rendre les étudiants conscients des différentes façons d'apprendre, de façon à enclencher chez eux le processus métacognitif de réflexion sur la façon dont ils apprennent le mieux [...]. Il revient ensuite aux enseignants de fournir aux étudiants des occasions d'apprendre de différentes façons. » La variété prend ici tout son sens.

Dans Form@sup, parmi les outils et méthodes qui apportent cette variété, citons l'usage du eLearning et d'un maximum de ses fonctionnalités ${ }^{10}$, le principe des séminaires virtuels, comprenant euxmêmes diverses modalités de travail ${ }^{11}$, la recherche thématique, les cours techniques avec défis en ligne et, enfin, le portfolio réflexif, qui permet de couvrir l'événement d'apprentissage ${ }^{12}$ non encore abordé dans les autres activités: la métacognition, ou la réflexion sur son propre apprentissage ${ }^{13}$. Inspirée des travaux de Schön (1983/1994) et d'Argyris (1993/1995), la pratique réflexive demandée à nos participants au travers de leur portfolio comprend, elle aussi, des sous-activités. Toutes sont orientées vers la réflexion sur l'enseignement, y compris la critique de Form@sup. Comme le fait remarquer Kathleen King (2004, p. 172), «impliquer des enseignants adultes dans la compréhension du pourquoi et $\mathrm{du}$ comment sont choisies et mises en œuvre les activités d'apprentissage amène les apprenants dans le cercle de responsabilité. » 
Les difficultés engendrées par cette volonté de variété sont multiples, essentiellement d'ordres pédagogique et organisationnel: une préparation longue, un encadrement important, une nécessité de rétroactions régulières, une énergie considérable investie dans la recherche permanente d'intervenants-témoins et une maintenance plus difficile du site en ligne.

Comme déjà signalé au point précédent, il est aussi parfois problématique de concevoir des activités qui soient variées en termes d'outils et d'événements d'apprentissages, mais qui soient aussi directement utiles au projet personnel de chaque enseignant. Le travail thématique, par exemple, est considéré comme trop éloigné de la préoccupation directe $\mathrm{du}$ projet (voir point 9 pour les options correctives prises en 2005-2006).

\section{Pourquoi une progression très encadrée?}

Avec Charlier et Peraya (2003, p. 204), nous voyons le tutorat comme

«l'ensemble des fonctions, des rôles et des tâches destinés à guider, aider et soutenir les apprenants engagés dans un système de formation partiellement ou entièrement à distance dans la réalisation de l'ensemble des activités individuelles ou collaboratives. Le tutorat porte sur les aspects d'apprentissage, mais aussi des aspects technologiques, relationnels et métacognitifs. »

Au sein de Form@sup, nous parlerons de tutorat pour les séminaires virtuels, les activités de forums et le travail thématique. Nous parlerons plutôt d'accompagnement pour le projet personnel.

Le tutorat est pratiqué au fil des diverses activités qui jalonnent Form@sup, mais aussi étudié en tant que tel, pour l'importance qu'il peut revêtir dans la motivation des étudiants et dans leur réussite. Partant du principe d'isomorphisme mentionné plus haut, nous pensons que ce qui est souhaitable pour les étudiants de nos participants l'est sans doute aussi pour eux-mêmes dans leur rôle d'apprenants au sein de Form@sup. L'encadrement est donc considéré comme une nécessité, un ferment du dispositif, l'un des ingrédients de son efficacité.

À titre d'introduction à leur article traitant $\mathrm{du}$ support aux enseignants, Brindley, Zawacki et Roberts (2003, p. 138) reprennent une phrase de Salmon (2002, p. 1) qui résume bien la nécessité du tutorat et l'aspect indispensable qu'il revêt dans l'apprentissage en ligne: "Indépendamment du degré de sophistication technologique, les apprenants en ligne ne souhaitent pas se passer de leurs encadrants humains ». Dans ce même ordre d'idée, Walti (2003, p. 237) mentionne une recherche menée par Ewing (2000, p. 213-214), qui remarque que :

«Si les applications des TIC dans l'apprentissage ne comprennent pas d'interaction visible et significative avec l'enseignant, il existe un véritable danger que l'apprenant ressente l'environnement éducatif comme étant significativement dépersonnalisé. [...] Même si cet aspect des TIC dans l'apprentissage prend un temps considérable au tuteur, il a été identifié comme étant un facteur de succès significatif. »

La distribution des rôles se fait par activité (séminaire virtuel / thèmes / accompagnement des projets / dossiers réflexifs / cours techniques) et non par fonction: chaque tuteur ou accompagnateur prend en charge une activité dans sa globalité et y cumule souvent, selon les catégories de Berge (1995), les rôles pédagogique (intellectuel et lié à la tâche), social (permettant de créer un environnement social favorisant l'apprentissage), managérial (tâches administratives, procédurales et organisationnelles) et technique (permettant aux participants d'être en confiance avec le système). 
Les difficultés rencontrées sont d'ordres humain, organisationnel et pédagogique : tout d'abord, l'accompagnement nécessite un accord minimal entre les personnes, au service du projet. Cet accord nécessite souvent des mises au point et, parfois, l'ajout d'un accompagnateur additionnel, comme modérateur du processus.

Ensuite, il faut limiter le volume du tutorat et de l'accompagnement et en préciser les contours, sous peine de voir certains participants attendre que les accompagnateurs développent leur projet à leur place. Résister à ce type de pression demande un cadre qui le prévoit expressément.

Enfin, il faut assurer la formation de ces tuteurs et accompagnateurs. Outre les colloques, conférences, lectures, inclusions dans des communautés de pratiques ou autres moyens de formation individuelle des membres de l'équipe, nous avons mis au point une formation participante interne, destinée à combler ce que l'équipe estime être ses manques. Les compétences majeures à acquérir ont été isolées et le LabSET organise une séance de trois heures chaque mois pour augmenter le niveau de professionnalité de l'équipe d'encadrement. Cet investissement interne sur le long terme nous paraît indispensable.

\section{Pourquoi une évaluation des performances documentée, continue et variée?}

Entre autres caractéristiques, l'évaluation des performances pratiquée dans le dispositif Form@sup est documentée, continue, fréquente, variée et de complexité croissante. L'évaluation est précisément ce qui suscite chez nos participants le plus de commentaires et de réflexions critiques. L'équipe encadrante est elle-même en questionnement continu par rapport à cette évaluation, aucune décision n'étant parfaitement en accord avec tous nos principes. L'évaluation, au sein de Form@sup, a d'ailleurs varié d'année en année, indice qu'il s'agit là d'un point très critique du fonctionnement d'un dispositif de formation.

Quelques principes de base président à nos choix de ces types d'évaluation. Nous retiendrons principalement la documentation ${ }^{14}$, la continuité et la variété. Nous trouvons important que toutes les notes soient communiquées aux participants assorties de commentaires, de façon à leur permettre d'en tenir compte et de s'améliorer de façon continue, que ce soit dans les séminaires virtuels, dans les travaux thématiques ou dans le projet personnel. Tous les critères utilisés pour juger de ces différentes performances sont communiqués en ligne en début d'année, dans le "guide illustré de la formation ». La continuité ou la répétition permet aux participants de se voir progresser et de remédier aux faiblesses diagnostiquées. La diversité des types d'évaluation, tout comme illustré plus haut à propos des méthodes d'enseignement, permet de faire vivre aux participants un maximum de situations par rapport auxquelles ils devront se positionner en tant qu'enseignant dans leur propre cours.

Pour décrire ces évaluations de façon plus précise, nous dirons que leur référence est souvent critérielle, leur résultat diagnostic et leur visée formative (même si souvent aussi sanctionnante). L'évaluation est souvent centrée sur le produit (une seule activité évalue spécifiquement le processus) et pluridimensionnelle. Sa cible est toujours individuelle et privée, ses opérateurs sont majoritairement des experts, souvent externes, même si deux activités comportent de l'autoévaluation. Elle est tantôt ponctuelle, tantôt continue ou répétée (évaluation du cours grâce à la grille de critères de qualité), souvent améliorable, à source subjective (objective dans deux activités seulement), à contrat négocié et procédure adaptative. Les critères d'évaluation sont toujours annoncés. 
La principale difficulté est ici de concilier variété des pratiques évaluatives et concordance entre ces pratiques et les compétences dont nous visons le développement. Par exemple, faire vivre une modalité comme le QCM testant la compréhension de principes n'est pas directement nécessaire pour développer les compétences visées dans Form@sup, mais est utile à la réflexion sur son propre cours en ligne. Or, si un minimum d'enseignants ne l'ont pas vécu, les échanges au sein du groupe auront peu de richesse. La tentation est donc grande de rendre obligatoires une multitude d'activités... ce qui est en désaccord avec notre vision de la professionnalisation des enseignants basée sur une pratique réflexive plus que sur un amoncellement d'obligations. Comme le dit Biggs (1999, cité dans Weimer, 2002, p. 15), «ce que les étudiants travaillent et leur façon de travailler dépendent majoritairement de la façon dont ils imaginent qu'ils vont être évalués. Les pratiques d'évaluation doivent envoyer les bons signaux...». Envoyer les bons signaux est complexe, ce point est sans doute le plus critique dans notre dispositif actuel.

Un élargissement de la communauté apprenante à des participants qui ne font pas partie de Form@sup est peut-être l'une des clés de ce problème. Dans ce cas, pour certains types d'activités comme les discussions, échanges de pratiques ou débats d'idées, chaque enseignant trouverait plus facilement des partenaires pour les problématiques qu'il aurait déterminées comme nécessaires à son développement professionnel propre. Nous tenterons cette expérience moins contraignante en 2005-2006.

\section{Pourquoi une régulation permanente?}

Un dispositif efficace est aussi un dispositif qui vit au gré des avis instruits de tous ses acteurs. Ces avis sont issus des dossiers réflexifs, des entretiens avec les acteurs et de questionnaires ponctuels. Ils sont complémentés par l'analyse des interactions dans les forums, les chats et différents outils de communication interne et par la très importante analyse des connexions et des trajectoires des participants en ligne, reflet de leur activité et indicateur de leurs difficultés.

Form@sup est un dispositif jeune. Depuis sa mise en route en 2001, chaque année a vu des refontes de contenus, des modifications méthodologiques, des clarifications d'interfaces et des changements significatifs dans les rôles des tuteurs et accompagnateurs ou dans la façon de considérer son évaluation. Au fil du temps, une part de plus en plus large du dispositif a été prévue en ligne, la notion de communauté virtuelle ${ }^{15}$ a pris sens grâce aux séminaires virtuels ${ }^{16}$, les activités ont été de plus en plus logiquement articulées autour de modèles structurants, une ouverture vers l'étranger s'est dessinée clairement et des partenariats ont pu être envisagés avec sérénité.

Les changements ne sont pas pour autant derrière nous. La régulation est permanente. Parmi les changements opérés en 2005-2006, soulignons la refonte des compétences à acquérir, influencée par des associations professionnelles comme HERDSA $^{17}$, POD $^{18}$ et SEDA $^{19}$ ainsi que par des modèles issus du courant de professionnalisation de l'enseignement comme celui de Trigwell, Martin, Benjamin et Prosser (2000), que nous avons adapté à nos besoins. En conséquence, trois productions majeures sont demandées aux participants :

- Un rapport de «recherche en classe ${ }^{20}$ » nourri par la littérature pédagogique ${ }^{21}$. Cette recherche remplace le travail thématique, de façon à mieux s'articuler avec le projet personnel de chacun. Le traitement de la question de recherche vient naturellement alimenter les décisions «instructionnelles » ou didactiques de chacun dans son propre cours; 
- Le développement d'un projet personnel (comme les années précédentes, son propre cours en ligne ou son projet APP/PBL). Nous y introduisons la gestion en ligne de la qualité des projets (Georges et Van de Poël, 2005) et proposons désormais aux participants de décider eux-mêmes des moments d'évaluations de ces qualités;

- La constitution d'un portfolio d'enseignement, qui englobe les deux premières productions et demande à chacun un point réflexif sur sa pratique (voir plus bas).

Selon la classification des types de portfolio de van Tartwijk, Driessen et EPICC Team (2005), celui de Form@sup est de type combiné, avec un accent tout particulier sur la pratique réflexive, mais comportant également les nécessaires composantes de présentation (pour rendre possible un partage d'expériences, les collègues doivent en savoir un minimum sur le contexte de chacun) et de développement (pour les mêmes raisons de partage, puisque chacun explore des questions de recherche différentes, ces trajets individuels doivent être précisés). Pour permettre au lecteur de s'en faire une idée très précise, nous joignons en annexe la description du «portfolio d'enseignement» que doit constituer chaque participant de Form@sup. Ce portfolio fait l'objet d'une attention toute particulière dans Form@sup car, outre ses fonctions de guide réflexif et d'outil d'évaluation au sein même de la formation, nous le considérons également comme un atout pour l'avancement professionnel et la valorisation de nos enseignants participants. La constitution, début 2005 à l'Université de Liège, de l'Institut de formation et de recherche en enseignement supérieur (IFRES) pourrait faciliter cette valorisation des portfolios d'enseignement dans la carrière académique.

La consolidation du dispositif par son ancrage dans la recherche et dans les avancées des organisations professionnelles (SEDA, HERDSA, POD), sa flexibilisation et sa modularisation au travers de l'outil de gestion de la qualité des projets nous semblent susceptibles d'augmenter la motivation des participants, mais aussi de renforcer la cohérence de notre dispositif avec le courant de professionnalisation de l'enseignement dans le supérieur et celui de la validation des acquis de l'expérience.

Comme le soulignent Cottrell et Jones (2003, p. 178) dans une étude réalisée aux États-Unis, sur 47 instructeurs issus de 4 universités,

«tous les instructeurs ont réalisé qu'une composante essentielle du professionnalisme de l'enseignement/apprentissage réside dans l'usage des résultats d'évaluation pour opérer des changements délibérés au sein du cours de façon à maximiser l'apprentissage et le développement des étudiants. »

Form@sup n'échappe pas à cette constatation. La réflexion sur la régulation du dispositif constitue une part cruciale du professionnalisme de tous ses acteurs, qu'ils soient encadrants ou participants.

\section{Discussion}

Au moment où les universités et les Hautes Écoles européennes tentent de revisiter leur pédagogie pour faire face aux nouveaux défis de l'internationalisation croissante et des exigences de la convention de Bologne, le courant international de professionnalisation de l'enseignement dans le supérieur propose des modèles et illustrations susceptibles d'aider ces institutions à améliorer la qualité de leurs enseignements. Wulff et Austin (2004, p. 269) constatent que: «Des universités renommées, orientées recherche, et des associations professionnelles, intègrent les principes du mouvement de professionnalisation de l'enseignement et de l'apprentissage dans le développement de programmes professionnels spécifiques avec pour but de préparer les futurs enseignants. » 
Parallèlement, le déploiement de l'eLearning fait son chemin, à grands pas. Une offre performante de formation en ligne vient désormais enrichir les catalogues de formation initiale et de formation continuée. Au niveau européen, des programmes de financement communautaires et divers plans d'action ont été mis en place par la Commission européenne. En Belgique, des cours en ligne de qualité sont produits grâce à des initiatives régionales ou individuelles.

Form@sup participe à ces élans conjoints, utilisant l'eLearning pour favoriser le questionnement et l'outillage de la pratique professionnelle des participants, au bénéfice de la qualité des enseignements. La pédagogie universitaire est en effet aujourd'hui une réelle discipline, avec sa rigueur, son ancrage scientifique et ses études d'impact.

Form@sup souhaite favoriser, chez les professionnels de la formation, non seulement une réflexion, mais aussi une réelle capacité à développer des environnements nouveaux susceptibles de rehausser la formation universitaire et de démocratiser les savoirs fondamentaux. Nous soutenons l'affirmation de George Lueddeke (2003, p. 216) selon laquelle "globalement, les approches de professionnalisation de l'enseignement partagent aujourd'hui l'objectif général d'améliorer l'apprentissage des étudiants. »

Form@sup, après quatre ans, est toujours en pleine évolution. De nombreuses difficultés ont été surmontées, d'autres persistent. Nous espérons que cette formation ne cessera d'évoluer et qu'elle relèvera ainsi les défis que nous proposeront encore demain les nombreuses facettes de la profession enseignante.

\section{Références}

Allen, M., (2003). Using scoring rubrics. Récupéré le 10 septembre 2005 du site de la California State University, Division of Academic Affairs, section
Student learning outcomes - Scoring rubrics, http:// www.calstate.edu/acadaff/sloa/links/using rubrics.shtml

Allinson, C. W. et Hayes, J. (1996). The Cognitive Style Index: A measure of intuition-analysis for organisational research. Journal of Management Studies, 33, p. 119-135.

Argyris, C. (1995). Savoir pour agir. Surmonter les obstacles à l'apprentissage organisationnel. Paris: Interéditions. (Ouvrage original publié en 1993 sous le titre Knowledge for action. A guide to overcoming barriers to organizational change. San Francisco: Jossey-Bass.)

Berge, Z. L. (1995). Facilitating computer conferencing: Recommendations from the field. Educational Technology, 35(1), p. 22-30. (Version différente récupérée le 10 septembre 2005 du site eModerators, http:/ / www.emoderators.com/moderators / teach_o $\underline{\text { nline.html) }}$

Bernath, U. et Rubin, E. (dir.) (2003). Reflections on teaching and learning in an online master program. A case study. Oldenburg, Allemagne : Bibliotheks- und Informationssystem der Universität Oldenburg.

Boice, R. (2000). Advice for new faculty members: Nihil nimus. Needham Heights, MA : Allyn and Bacon.

Boyer, E. L. (1990). Scholarship reconsidered: Priorities of the professoriate. Princeton, NJ : Princeton University Press, Carnegie Foundation for the Advancement of Teaching.

Brindley, J., Zawacki, O. et Roberts, J. (2003). Support services for online faculty: The provider and the user perspectives. Dans U. Bernath et E. Rubin (dir.), Reflections on teaching and learning in an online master program. A case study (p. 137-165). Oldenburg, Allemagne : Bibliotheks- und Informationssystem der Universität Oldenburg.

Brown, R. et Donovan, C. (2000). The relationship between research and teaching in higher education: Present realities, future possibilities (Rapport d'un séminaire organisé conjointement par le 
Southampton Institute et le Higher Education Funding Council). Southampton, Royaume-Uni : Southampton Institute.

Charlier, B. et Peraya, D. (2003). Technologie et innovation en pédagogie. Dispositifs innovants de formation pour l'enseignement supérieur. Bruxelles : De Boeck et Larcier.

Coffield, F., Moseley, D., Hall, E. et Ecclestone, K. (2004). Learning styles and pedagogy in post-16 learning: A systematic and critical review. London: Learning and Skills Research Centre. Récupéré le 10 septembre 2005 du site de la Learning and Skills Development Agency, http://www.lsda.org.uk / files/pdf/1543.pdf

Cottrell, S. A. et Jones, E. A. (2003). Researching the scholarship of teaching and learning : An analysis of current curriculum practices. Innovative Higher Education, 27(3), p. 169-181. Récupéré le 10 octobre 2006 du site de la revue, http:/ / www.springerlink.com/content/h027h322h6 $\underline{753704 / \text { fulltext.pdf }}$

Delfosse, C., Harmeling, D., Poumay, M. et Leclercq, D. (2003, janvier). Les facteurs de succès dans l'accompagnement $d u$ portage à distance de cours. Expérience du LabSET. Communication présentée aux $2^{\mathrm{e}}$ Rencontres du Kirchberg. Colloque scientifique sur les pratiques de téléformation, Luxembourg (L).

Entwistle, N. J., McCune, V. et Walker, P. (2001). Conceptions, styles and approaches within higher education: Analytic abstractions and everyday experience. Dans R. J. Sternberg et L.-F. Zhang (dir.), Perspectives on thinking, learning and cognitive styles (p. 103-136). Mahwah, NJ : Lawrence Erlbaum.

Ewing, J. (2000). Enhancement of online and offline student learning. Educational Media International, 37(4), p. 205-217.

Gagne, R. M. et Briggs, L. J. (1974). Principles of instructional design. New York: Holt, Rinehart and Winston.
Georges, F. et Van de Poël, J. F. (2005). Évaluations d'un cours en ligne : produit, usage et impact. Dans N. Rege Colet (dir.), Actes $d u 22^{e}$ congrès de l'Association internationale de pédagogie universitaire. L'enseignement supérieur $d u 21^{e}$ siècle : de nouveaux défis à relever [CD ROM]. Genève, Suisse: Association internationale de pédagogie universitaire.

Herrmann, N. (1996). The whole brain business book. New York : McGraw-Hill.

Honey, P. et Mumford, A. (2000). The learning styles helper's guide ( $1^{\mathrm{e}}$ éd.). Maidenhead, Royaume-Uni : Peter Honey.

Kelly, K. (2005, 21 juin). Re: Personalize Moodle for individual learning style. Message publié dans le forum Teaching strategies du cours Using Moodle. Récupéré le 10 juillet 2005 du site du forum, http: / / moodle.org/mod/forum/ discuss.php?d=260 $\underline{66 \& \text { parent }=123089}$

King, K. P. (2004). Both sides now: Examining transformative learning and professional development of educators. Innovative Higher Education, 29(2), p. 155-174. Récupéré le 17 octobre $2006 \mathrm{du}$ site de la revue, http:/ / springerlink.metapress.com/content/g27701 $\underline{47 n 1 k 1 r 127 / \text { fulltext.pdf }}$

Kolb, D. A. (1984). Experiential learning: Experience as the source of learning and development ( 1 éd.). Englewood Cliffs, NJ : Prentice Hall.

Kolb, D. A. (1999). The Kolb Learning Style Inventory, Version 3. Boston, MA: Hay Group, Hay Resources Direct.

Kreber, C. (2002). Teaching excellence, teaching expertise, and the scholarship of teaching. Innovative Higher Education, 27(1), p. 5-23. Récupéré le 17 octobre $2006 \mathrm{du}$ site de la revue, http:/ / springerlink.metapress.com/content/g4p670 700rv77017/ fulltext.pdf

Lambert, L. M. et Tice, S. L. (dir.) (1993). Preparing graduate students to teach. A guide to programs that 
improve undergraduate education and develop tomorrow's faculty. From a comprehensive national survey of teaching assistant training programs and practices. Washington, DC : American Association for Higher Education.

Laurillard, D. (2002). Rethinking university teaching. A conversational framework of the effective use of learning technology ( $2^{\mathrm{e}}$ éd.). London : Routledge Falmer.

Leclercq, D., Conti, C., DeKetele, J.-M., Delhaxhe, M., Dupont, P., Lambert, J.-P., Lambotte, J.-P., Noel, B., Romainville, M. et Wolfs, J.-L. (2003). Le projet MOHICAN - Check-up : son origine, ses intentions, ses méthodes. Dans D. Leclercq (dir.), Diagnostic cognitif et métacognitif au seuil de l'université. Le projet MOHICAN-CIUF (p. 13-31). Liège, Belgique : Editions de l’Université de Liège.

Leclercq, D. (2005). Édumétrie et docimologie pour praticiens chercheurs. Liège, Belgique: Éditions de l’Université de Liège.

Leclercq, D. et Poumay, M. (2004). Une définition opérationnelle de la métacognition. Dans A. Chiadli (dir.), Actes $d u \quad 21^{e}$ congrès de l'Association Internationale de Pédagogie Universitaire. L'AIPU : 20 ans de recherche et d'actions pédagogiques; bilans et perspectives [CD ROM]. Marrakech, Maroc: Association internationale de pédagogie universitaire.

Leclercq, D. et Poumay, M. (2005). The 8 learning events model and its principles (version 2005-1). Récupéré le 10 septembre 2005 du site du Laboratoire de soutien à l'enseignement télématique (LaBSET), http:/ / www.labset.net/media/prod/8LEM.pdf

Lueddeke, G. R. (2003). Professionalising teaching practice in higher education: A study of disciplinary variation and 'teaching-scholarship'. Studies in Higher Education, 28(2), p. 213-228.

Mullinix, B. B. (2004, mise à jour mai 2005). Rubrics. Récupéré le 10 septembre 2005 du site de Monmouth University, Faculty Resource Center, http: / /its.monmouth.edu/facultyresourcecenter/ru brics.htm

Poumay, M. (2003). Keys to promote good practices in ODL by a TECCC approach (Training Embedded Coached Course Construction): Illustrations through a postgraduate degree and an annual competitive call. Educational Media International, 40(3-4), p. 229237

Poumay, M. (2007). Pour professionnaliser le métier d'enseignant du supérieur: le Master complémentaire Form@sup. Revue internationale des technologies en pédagogie universitaire, 3(2), p. 3-13.

Salmon, G. (2002, avril). Hearts, minds and screens: Taming the future. Discours d'ouverture présenté au Education Electronic Commerce Action Team (EduCAT) Summit: Innovations in e-Education, Hamilton, New Zealand. Récupéré le 10 septembre $2005 \mathrm{du}$ site All Things in Moderation, section Research, Presentations to 2002, http://www.atimod. $\mathrm{com} /$ research/presentations/Hearts3.htm

Sanders, W. B. (2001). Creating learning-centered courses for the World Wide Web. Needham Heights, MA : Allyn and Bacon.

Schön, D. (1994). Le praticien réflexif. À la recherche du savoir caché dans l'agir professionnel (J. Heynemand et D. Gagnon, trad.). Montréal : Éditions Logiques. (Ouvrage original publié en 1983 sous le titre The Reflective Practitioner. New York : Basic Books).

Sternberg, R. J. (2001). Epilogue: Another mysterious affair at styles. Dans R. J. Sternberg et L.-F. Zhang (dir.), Perspectives on thinking, learning and cognitive styles (p. 249-252). Mahwah, NJ : Lawrence Erlbaum.

Trigwell, K., Martin, E., Benjamin, J. et Prosser, M. (2000). Scholarship of teaching: A model. Higher Education Research and Development, 19(2), p. 155-168.

van Tartwijk, J., Driessen, E. et EPICC Team (2005, 2 mars). Typologie de portfolios électroniques. Récupéré le 19 octobre 2006 de l'ancien site Insight: Knowledge base for new technology and education (du European Schoolnet), section School practice, 
français, http://insight-old.eun.org/eun.org2/eun/ $\underline{\mathrm{fr} / \text { Insight SchoolPractice/ content.cfm?ov=33515\&la }}$ $\underline{\mathrm{ng}}=\mathrm{fr}$ (Document original publié le 26 avril 2004 sous le titre Exploring different types of electronic portfolios. Récupéré de la section anglaise du même site.)

Vermunt, J. D. (1994). Inventory of learning styles in higher education. Tilburg, Pays-Bas : University of Tilburg, Department of Educational Psychology.

Viau, R. (1994). La motivation en contexte scolaire. Québec, Canada : Éditions du renouveau pédagogique.
Walti, C. (2003). Experiencing a new paradigm: Elements, aspects, and structure of selected courses in the MDE Program. Dans U. Bernath et E. Rubin (dir.), Reflections on teaching and learning in an online master program. A case study (p. 227-243). Oldenburg, Allemagne : Bibliotheks- und Informationssystem der Universität Oldenburg.

Weimer, M. (2002). Learner-centered teaching: Five key changes to practice. San Francisco : Jossey-Bass.

Wulff, D. H., Austin, A. E. (dir.) (2004). Paths to the professoriate: Strategies for enriching the preparation of future faculty. San Francisco : Jossey-Bass. 


\section{Annexe. Le portfolio d'enseignement de Form@sup}

\begin{tabular}{|c|c|}
\hline & Portfolio d'enseignement de Form@sup \\
\hline \multirow[t]{5}{*}{ Présentation } & Votre vision de l'enseignement et vos buts en tant qu'enseignant \\
\hline & $\begin{array}{l}\text { Vos cours, vos TPs et vos différentes charges en rapport avec l'enseignement } \\
\text { (y compris différents comités) }\end{array}$ \\
\hline & $\begin{array}{l}\text { Vos formations en matière d'enseignement : diplômes, ateliers, conférences, séminaires, } \\
\text { accompagnements individualisés, communautés de pratiques, etc. }\end{array}$ \\
\hline & $\begin{array}{l}\text { Vos présentations ou publications à caractère pédagogique (si trop nombreuses, choisir les plus } \\
\text { significatives) }\end{array}$ \\
\hline & $\begin{array}{l}\text { (Optionnel) Les avis de votre entourage sur votre enseignement : vos étudiants, vos collègues, votre } \\
\text { président de département, etc. }\end{array}$ \\
\hline \multirow[t]{4}{*}{$\begin{array}{l}\text { Réflexion- } \\
\text { Intervention }\end{array}$} & $\begin{array}{l}\text { Vos efforts pour améliorer votre enseignement : résumé de ce que vous considérez comme les } \\
\text { changements les plus marquants dans votre pratique enseignante (errements et tournants) }\end{array}$ \\
\hline & $\begin{array}{l}\text { Un exemple approfondi : la Réflexion-Intervention menée dans le cadre de Form@sup. Adoptez un } \\
\text { point de vue critique en présentant les principales forces de votre intervention (votre cours en ligne, } \\
\text { votre développement APP, etc.). Si cela se justifie, joignez éventuellement en annexe une copie du } \\
\text { détail des qualités de votre produit. }\end{array}$ \\
\hline & $\begin{array}{l}\text { Au départ d'une question de recherche, votre spécialisation dans un thème lié à l'enseignement (essai } \\
\text { ou article scientifique). Cette réflexion doit : } \\
\text { Poser un problème et justifier en quoi il est porteur pour l'apprentissage } \\
\text { Démontrer une bonne connaissance du domaine choisi, notamment par référence à la littérature } \\
\text { scientifique } \\
\text { Montrer en quoi ces références ont été mobilisées dans votre travail } \\
\text { Évaluer l'impact de votre action (exigence : niveau } 2 \text { de Kirkpatrick) } \\
\text { Ouvrir vers des perspectives/orientations / questions }\end{array}$ \\
\hline & $\begin{array}{l}\text { Brièvement, reprenant éventuellement des données utilisées plus haut, quelques indicateurs de } \\
\text { qualité de votre enseignement (voir la grille HERDSA) : } \\
\text { Une preuve que votre enseignement prend en compte l'apprentissage de vos étudiants } \\
\text { Une preuve que vos évaluations encouragent/ soutiennent l'apprentissage de vos étudiants } \\
\text { Une preuve que vos avancées pédagogiques se font sur la base de différents types de données, dont } \\
\text { des données objectives } \\
\text { Une preuve démontrant une prise en compte particulière (et efficace) de minimum } 3 \text { autres des } 47 \\
\text { critères de la grille de bonne pratique HERDSA }\end{array}$ \\
\hline Perspectives & Vos questions en suspens et prochains pas prévus en matière de développement professionnel \\
\hline \multirow[t]{2}{*}{$\begin{array}{l}\text { Communication } \\
\text { publique }\end{array}$} & $\begin{array}{l}\text { Date, lieu et public cible de la présentation orale de certains des éléments ci-dessus (au choix), } \\
\text { mentionnant minimalement la question de recherche }\end{array}$ \\
\hline & Brefs commentaires sur cette présentation \\
\hline
\end{tabular}




\section{Notes}

${ }^{1}$ L'option APP, organisée par les bureaux pédagogiques de la Faculté de médecine, ne sera pas abordée ici.

${ }^{2}$ Par « cours à distance », nous entendons ici un dispositif de formation utilisant : (1) un ensemble d'acteurs (professeurs, tuteurs, moniteurs), (2) des activités d'apprentissage, (3) des outils de communication (tels courriels, forums de discussion, groupes de travail), (4) une série de médias et supports (tels notes de cours, images fixes ou animées, séquences sonores, etc.), (5) une plate-forme intégrée d'enseignement distribué, ... le tout faisant usage de stratégies variées alliant souvent le présentiel et la distance en fonction des objectifs visés et des contraintes du projet. Nous lui préférerons souvent l'expression « cours en ligne », qui exclut les dispositifs basés sur l'envoi postal de documents, et utiliserons le terme «eLearning » pour désigner l'activité de l'utilisateur dans un tel dispositif en ligne.

${ }^{3}$ Projet DéCLIC, qui regroupe plusieurs grands acteurs wallons de la formation. Voir http: / www.equal-declic.net/ ${ }^{4}$ FORMADIS pour FORMAtions à DIStance, voir http:/ / www w.labset.net/formadis/. Dans ce projet, le LabSET lance chaque année un appel à propositions et sélectionne avec l'ULB, son partenaire, 10 à 15 organisations qui seront accompagnées pendant une année dans le portage en ligne de leur premier cours, ceci avec un accent sur des critères de qualité. Ce projet est subventionné par le Fonds Social Européen, le Ministère de l’Emploi et de la Formation Professionnelle de la Région Wallonne et le Ministère de l'Enseignement Supérieur et de la Recherche de la Communauté Française de Belgique.

${ }^{5}$ Voir http: / / www.pmtic.net/. Ce « Plan mobilisateur TIC » est coordonné par le LabSET.

${ }^{6}$ Nous remarquons cependant que même des enseignants chevronnés sont intéressés par une telle formation. Leur présence en formation est d'ailleurs très appréciée par leurs collègues.

${ }^{7}$ L'option APP/PBL, coordonnée par le bureau pédagogique de la Faculté de médecine de l'ULg, n'est pas abordée dans cette analyse.

${ }^{8}$ Le terme « design instructionnel » ne fait pas partie de la langue française, mais il nous semble bien approprié à notre action, car il limite le projet à une réflexion didactique.

9 « Les cours de qualité fondent leurs objectifs sur une bonne analyse des besoins des apprenants; ils incluent des composants motivationnels (affectifs) aussi bien que cognitifs et métacognitifs, utilisent des méthodes choisies sur la base de théories valides et explicites, évaluent les produits et processus d'apprentissage par des méthodes et des outils adéquats et écologiquement valides, débouchant sur des indices diagnostiques et porteurs de sens pour les apprenants. Ils permettent par ailleurs à une large majorité d'entre eux d'atteindre les objectifs d'apprentissage fixés, ceci avec un haut niveau de maîtrise. » (Poumay, 2003, p. 230).

${ }^{10}$ Nombreux outils de communication (en synchrone : chat et vidéoconférences; en asynchrone : forums, calendrier, portfolios, courriels, espaces de groupes, présentations personnelles, visualisation de sa progression), essentiels à la formation; espaces « contenus », à la fois pour structurer les contenus pédagogiques et techniques (textes, vidéos, présentations PowerPoint $(\subset$, nombreux liens internes et externes, glossaire, outils et grilles de travail pour les différentes étapes BEComeRIR, fiches d'activités, etc.) et pour présenter cette structure aux participants; tests formatifs et parfois sanctionnants, ponctuant les séminaires virtuels.

${ }^{11}$ Ils comprennent des travaux en groupes, d'autres individuels, des témoignages d'experts externes, des séminaires d'échanges entre participants, des séances présentielles et des travaux en ligne.

${ }^{12}$ Pour un éclairage sur les huit événements d'apprentissage, constituant le pendant des événements d'instruction de Gagne et Briggs (1974), voir Leclercq et Poumay (2005).

${ }^{13}$ Pour une définition opérationnelle de la métacognition, voir Leclercq et Poumay (2004).

${ }^{14}$ Ce principe correspond à ce que Leclercq (2005, p. 5 et 37) appelle «validité informative, ou diagnostique », qu'il décrit aux côtés de sept autres principes de validité des évaluations. 
${ }^{15}$ Telle que définie par l'Université Laval de Québec dans http: / / www.fse.ulaval.ca/fac/ tact/fr/html/prj-

7.1/ commune2.html : «Un groupe d'élèves et au moins un éducateur ou une éducatrice qui, durant un certain temps et animés par une vision et une volonté communes, poursuivent la maîtrise de connaissances, d'habiletés ou d'attitudes. »

${ }^{16}$ La littérature sur le sujet nous a influencés en ce sens. Citons par exemple Bernath et Rubin (2003, p. 12), qui notent que « ces séminaires se sont révélés être des communautés virtuelles de construction de connaissances ».

${ }^{17}$ HERDSA est la « Higher Education Research and Development Society » (voir http: / www.herdsa.org.au/), organisation professionnelle d'Australie et d'Asie australe qui offre un journal scientifique et de nombreuses ressources en ligne.

${ }^{18}$ POD est le « Professional and Organizational Development Network in Higher Education » (voir http: / / www.podnetwork.org/)

${ }^{19}$ SEDA est la «Staff and Educational Development Association » (voir http:/ / www.seda.ac.uk/), organisation professionnelle anglaise très active et proposant des formations et accréditations de différents niveaux : fellowship et associate fellowship.

${ }^{20}$ Le courant de la recherche en classe, né aux États-Unis au début des années 1990, a pour but d'aider les enseignants du supérieur à mieux comprendre ce que les étudiants retirent de leur cours, avec quelle qualité ils apprennent, quels sont les processus en œuvre et comment isoler les facteurs sur lesquels leur enseignement peut avoir une influence. Pour plus d'information, nous renverrons le lecteur à Allen (2003) et Mullinix (2004).

${ }^{21}$ Avec Brown et Donovan (2000, p. 14), nous sommes persuadée que pour donner à nos participants cette habitude d'ancrer leurs affirmations dans la recherche en éducation, il nous faut prévoir « l'introduction de la recherche dans le design des curricula, les étudiants eux-mêmes jouant un rôle clé dans la création des liens entre recherche et apprentissage. » 